\title{
Accretion-Outflow Activity in Herbig Ae/Be Stars
}

\section{Larisa Tambovtseva*}

Pulkovo Observatory of RAS, Pulkovskoe shosse 65, 196140 Saint Petersburg, Russian Federation

E-mail: lvtamb@mail.ru

\section{Vladimir Grinin}

Pulkovo Observatory of RAS, Pulkovskoe shosse 65, 196140 Saint Petersburg, Russian

\section{Federation}

E-mail: grinin@gao.spb.ru

\begin{abstract}
Young intermediate mass stars, so-called Herbig Ae/Be stars, demonstrate both accretion and outflow features in their emission spectra. Using non-LTE modeling of the hydrogen lines in the visible and near-infrared spectral regions we investigated a close vicinity of these stars and revealed the most important regions contributing to the line emission. The regions of the disk wind play the most important role in the radiation of the hydrogen lines. The regions with accreting matter contribute less to this radiation but can influence the shape of the line profiles. We conclude that a role of accreting processes may be different for the different stars. A radiation from the hot layers of accretion disks is small for the lines in the visible and negligible for the lines in the infrared spectral regions compared to other components surrounding Herbig Ae/Be stars.
\end{abstract}

Accretion Processes in Cosmic Sources - APCS2016

5-10 September 2016,

Saint Petersburg, Russia

* Speaker. 


\section{Introduction}

One of the best way to study physical and geometrical properties of the regions surrounding the young stars is to perform the non-LTE modeling of the radiation coming from these regions and forming emission spectra of the young stars. Reproduction of the emission lines in each component of the circumstellar envelope permits one to probe a closest vicinity of the young star unresolved with telescopes up to the present time.

Herbig Ae/Be stars (HAEBEs), as their low mass counterparts T Tauri stars (TTSs) follow principally the same evolutionary scenario. It is known that TTSs possess strong magnetic fields and well developed magnetospheres $\left(5 R_{*}-10 R_{*}\right)$. Their hydrogen emission spectra are formed mainly in the gas accreting onto the star within the funnels created by field lines of the magnetosphere (a magnetospheric accretion)(see, e.g., Hartmann et al. 1994; Muzerolle et al. 2001; Kurosawa et al. 2006). The hydrogen and helium lines, veiling, and ultraviolet radiation excesses successfully confirm a prediction of the star formation theory for TTSs.

Unlike T Tauri stars, it is not well known whether Herbig Ae/Be stars possess a well-developed magnetosphere. Their own magnetic fields are probably very weak: only several Herbig Ae stars (HAEs) with very weak magnetic fields on the order of a few hundred Gauss or weaker are known (Hubrig et al. 2007; Wade et al. 2009; Alecian et al. 2013). HAEs are rapid rotators, they are much more luminous, veiling in the spectra is absent. Hubrig et al. $(2011,2013)$ concluded that the magnetic field geometry can likely be described by a centered dipole with a polar magnetic field strength of several hundred Gauss.

Therefore, questions arise:

- Which components of the complex, multicomponent environment of intermediate mass Herbig Ae/Be stars are responsible for the line profiles?

- What is the role of the accreting regions in formation of the hydrogen emission lines?

- What kind of accretion is expected: magnetospheric accretion or boundary layer accretion?

- What is the ratio between the mass loss rate of the disk wind to the accretion rate?

To answer these questions, we considered three components of the environment of several Herbig Ae/Be stars: a magnetosphere, a disk wind, and an accretion disk. Three of five stars considered were supported with infrared (IR) interferometric observations.

\section{Accretion and outflow spectral signatures}

Young low-mass and intermediate-mass stars were studied in detail spectroscopically in all spectral ranges (see, e.g., Grinin et al. 2001; Beskrovnaya \& Pogodin 2004; Ismailov and Khalilov 2010; Grady et al. 2010; Eisner et al. 2010, 2015; Mendigutía et al. 2013). A close environment of the Herbig Ae/Be star is rather complex. Analyzing only one line profile one can hardly understand which mechanism prevails: accretion or outflow of the matter. A uniform picture for the line profile behavior does not exist even at the known inclination of the star plus disk system to the line of sight. The most powerful line in the spectra of young stars, $\mathrm{H} \alpha$ line, forms in the vast volume of 

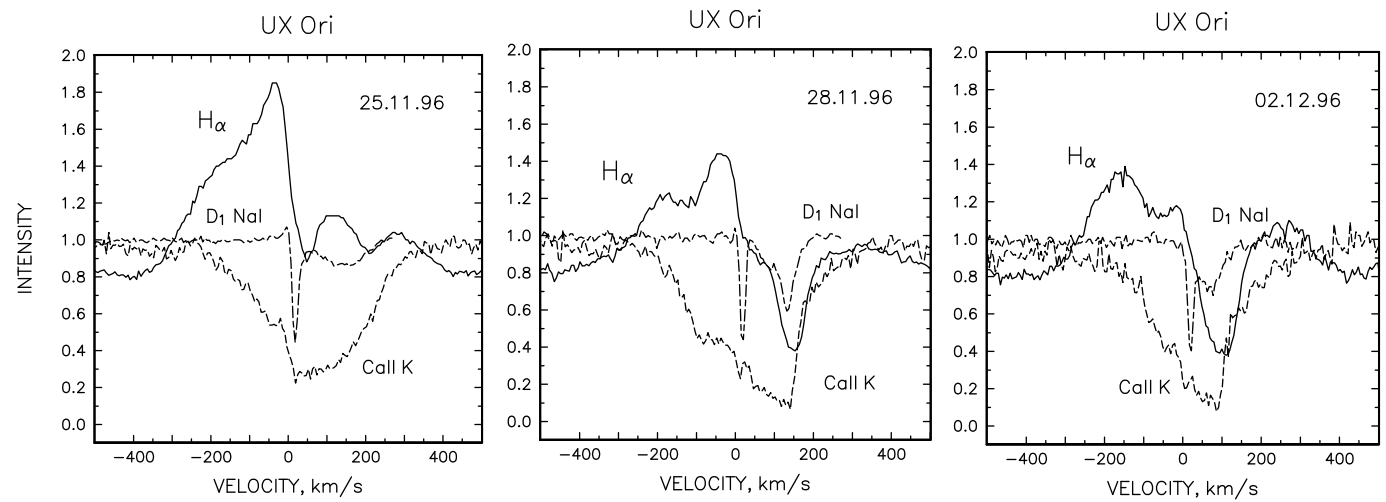

Figure 1: Profiles of $\mathrm{H} \alpha, \mathrm{D}_{1} \mathrm{NaI}$, and CaII K lines in the spectra of UX Ori obtained with NOT. An intensity of the line is normalized to an intensity of the continuum.

the circumstellar environment of the star. In some stars (e.g., AB Aur, MWC 480) this line as well as other Balmer lines permanently demonstrates a P Cygni type profile that is typical for the matter outflow. In the spectra of other stars this line has a single or double-peaked line profiles. At last, in the spectra of UX Ori type stars (UXORs) Balmer lines demonstrate accretion features. It is easy to understand keeping in mind that UXORs are a subclass of the young stars seen nearly edge-on (Grinin et al. 1991). For them accretion features in such lines as $\mathrm{H}, \mathrm{Ca}, \mathrm{Na}, \mathrm{Fe}, \mathrm{O}$ and others are clearly seen during the observations. One can see in Fig. 1 profiles of $\mathrm{H} \alpha, \mathrm{D}_{1} \mathrm{NaI}$, and CaII K lines in the spectra of UX Ori obtained with Nordic optical Telescopes (NOT) (Grinin et al. 2001). Moreover, the helium line HeI $5876 \AA$ is observed in HAEBEs; very often it has a shape which is typical for accretion of the matter onto the star. The presence of this line can be explained with the presence of the hot gas near the star surface. The gas can be heated when the matter falls onto the star and its kinetic energy transforms into the thermal one.

Non-LTE modeling of the different emission lines, namely, hydrogen, helium and sodium lines with the help of the various models of magnetospheric accretion has been performed by Tambovtseva et al. (1999, 2001), Muzerolle et al. (2004), and Mendigutía et al. (2011). However, none of existing model are able to reproduce the line profiles of the Balmer series having a Cygni shape. P Cygni type profiles are successfully modelled with the help of the magneto-centrifugal disk wind model developed by Blandford \& Payne (1982). A full description of the disk wind model is present in the paper by Grinin \& Tambovtseva (2011), a description of magnetospheric and boundary layer accretion models and results of modeling are present in the papers by Tambovtseva et al. 2014, 2016a; Grinin et al. 2016). When modeling the IR Br $\gamma$ line $(2.166 \mu \mathrm{m})$ together with interferometric observables, we considered both emitting regions: a magnetosphere and a disk wind. Results of modeling are given in the following section.

\section{Results of modeling}

During few last years we performed the non-LTE modeling of the Br $\gamma$ radiation from magnetospheric accretion region and the region of the disk wind for three Herbig Ae/Be stars: MWC 297, MWC 275 and HD 98922. Interferometric data were obtained with the VLTI. By means of VLTI/AMBER observations at high spectral resolution $(\mathrm{R} \sim 12000)$, we studied interferometric 
visibilities, wavelength-differential phases, and closure phases across the $\mathrm{Br} \gamma$ line of stars mentioned above. Our calculations showed that the $\operatorname{Br} \gamma$ emitting region cannot be so compact as the region of the magnetosphere which extends only up to $2-2.5$ stelar radii due to the rapid rotation of Herbig Ae/Be stars. However, it can provide a small emission source to the main contributor, magneto-centrifugal disk wind. Our modeling shows that the disk wind is the dominant contributor to the $\mathrm{Br} \gamma$ line compared to the magnetosphere and inner gaseous accretion disk (Weigelt et al. 2011; Garcia Lopez et al. 2015; Caratti o Garatti et al. 2015).
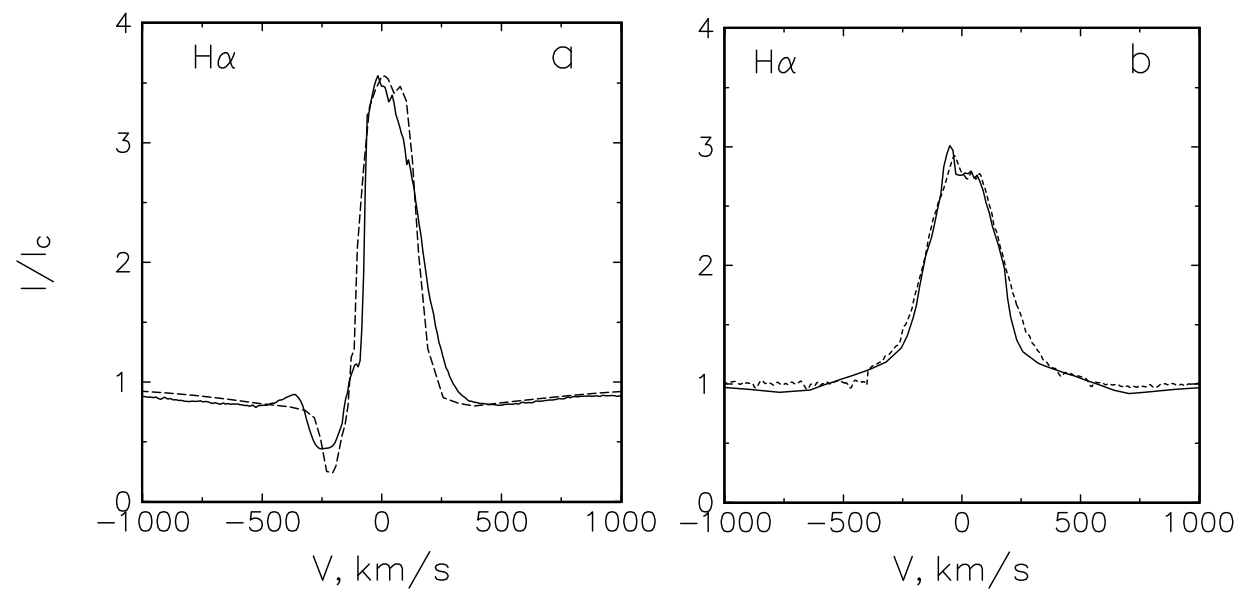

Figure 2: Calculated (solid line) and observed (dashed line) $\mathrm{H} \alpha$ line profiles in spectra of the stars: MWC 480 (a) and IL Cep (b). An intensity of lines $I$ is normalized to an intensity of the continuum $I_{c}$. See details in the text.

In the cases when spectroscopic observations were not supported with the interferometry we modelled several emission lines of the Balmer series (the stars MWC 480 and IL Cep) (Fig. 2). The observed $\mathrm{H} \alpha$ line profile for MWC 480 presented with the dashed line in Fig. 2a is the averaged one for several observational dates (full log of observations is published by Tambovtseva et al. 2016b). This line varies from night to night, nevertheless, the shape of the profile always has a classical P Cygni or P Cygni III type. The $\mathrm{H} \alpha$ line observed in the IL Cep spectrum is more stable (Fig 2b, dashed line). Theoretical profiles presented in Fig. 2 are obtained at inclinations $i$ within a range of $47^{\circ}-55^{\circ}$ for MWC 480 and $40^{\circ}-50^{\circ}$ for IL Cep $\left(i=0^{\circ}\right.$ at the face-on viewing). The model parameters are shown in the table.

It should be noted that the line profiles of the Balmer series are rather satisfactorily reproduced with the disk wind model without taking into account a radiation from the accretion zone. First of all, this points out to the fact that the radiation from this zone is significantly less than that from the disk wind region. Nevertheless, calculations of the emission of the UX Ori type stars seen nearly edge-on, show that under such angles (e.g., $70^{\circ}$ and more for VV Ser) it is problematic to reproduce the $\mathrm{Br} \gamma$ line profiles only in the framework of the disk wind model. The IR Br $\gamma$ line is more stable than lines in the optics. As our calculations showed (Tambovtseva et al. 2014), this line formed in the regions closest both to the star and to the surface of the accretion disk. Usually, in HAEBEs the line profiles of this line are narrow and single (Kraus et al. 2008). This implies that in the regions of the line formation the gas does not have high radial and tangential velocities.

What a region is able to form a narrow line with a low intensity? The radiation from such 


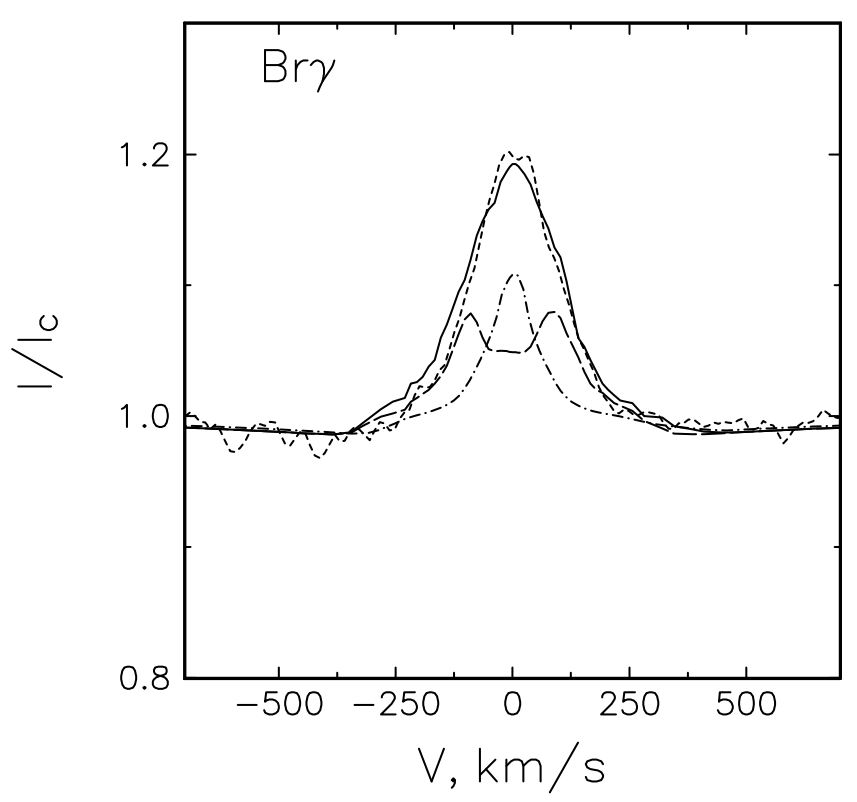

Figure 3: The star VV Ser. The Br $\gamma$ line profiles forming in the magnetosphere (dot-dashed line), from the disk wind (long dashed line). The total profile is shown with a solid line and the observed one with a short dashed line. An intensity of lines $I$ is normalized to an intensity of the continuum $I_{c}$. Inclination is $70^{\circ}$.

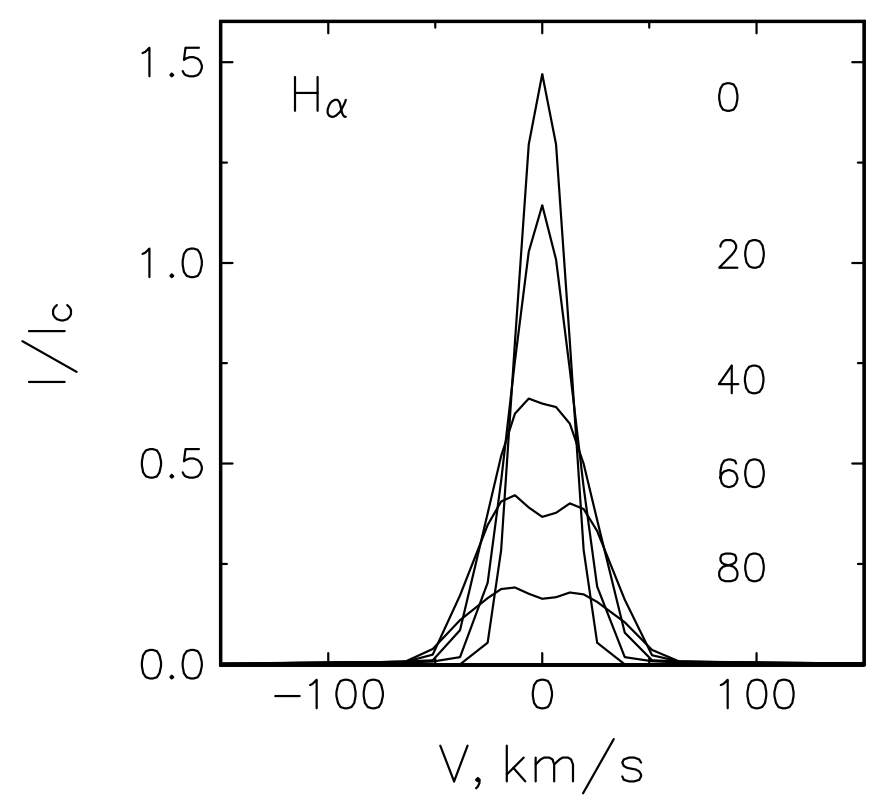

Figure 4: The $\mathrm{H} \alpha$ line profiles forming in hot surface layers of accretion disk. Inclinations are from pole-on (the narrowest profile) to $80^{\circ}$ (the widest one). An intensity of lines $I$ is normalized to an intensity of the continuum $I_{c}$. 
a region could complete a gap in the double-peaked profile originating in the disk wind region at such inclinations. It may be 1) a bi-polar outflow of the almost non-rotating gas, 2) a remote photoevaporating disk wind, and 3) an accretion of the matter onto the stars in the pole regions restricted with thin cones. Physical mechanism of the first outflow cannot be easily explained because in the young stars they are not powerful (e.g., Cranmer 1998), Zanni \& Ferreira 2009,2013). The second type of outflow is physically understood and well known but, as our calculations show (Tambovtseva et al, 2016a), the $\mathrm{Br} \gamma$ radiation is not enough to fill in the central gap in the line profile. The third mechanism is physically well-founded; it produces a quite noticeable narrow line. However, such a narrow line points out to that the falling gas practically does not rotate. This result leads to a hypothesis of a certain structure of the stellar magnetic field: an inclined dipole. The calculated $\mathrm{Br} \gamma$ line profile in the spectrum of VV Ser is shown in Fig. 3. The line profiles originating in the different components of the star environment are shown with the different line types. The short-dashed line refers to the observed line profile. The disk wind model parameters are present in the table. The following parameters are present: boundaries of the disk wind launching regions $\omega_{1}-\omega_{N}$ where $\omega$ is a cylindric radius in the units of the stellar radii; a half-opening angle $\theta_{1}$; the ratio of the gas terminal velocity to the Keplerian velocity at the footpoint of a streamline (SL) which can be constant for each SL or changes from one SL to another; the mass ejection parameter $\gamma$ which permits us to distribute the matter among streamlines in a different way; the mass loss rate expressed in units of $M_{\odot}$ per year, an acceleration parameter $\beta$ in the radial velocity law, and the gas temperature. Parameters of the accretion models are as follows: the gas flows from the outer radius $R_{\text {out }}=3 R_{*}$ to the star surface within two cones having half-opening angles $\theta_{\text {in }}$ and $\theta_{\text {out }}$ equaled correspondingly to $10^{\circ}$ and $30^{\circ}$. The electron temperature $T_{e}$ changes with the distance $r$ as

$$
T_{e}(r)=T_{e}\left(R_{*}\right)\left(r / R_{*}\right)^{-\alpha}
$$

where $\alpha=1 / 3$ and the gas temperature the star surface $T_{e}\left(R_{*}\right)=8000 \mathrm{~K}, R_{*}$ the stellar radius. The accretion rate was chosen as $3 \times 10^{-7} M_{\odot} \mathrm{yr}^{-1}$. A detail description of modelling one can find in the paper by Tambovtseva et al. (2016a).

\begin{tabular}{|c|c|c|c|c|c|c|c|}
\hline Star & $\omega_{1}-\omega_{N}$ & $\theta_{1}$ & $v_{\infty} / u_{K}$ & $\gamma$ & $\dot{M}_{w}$ & $\beta$ & $T$ \\
\hline & $R_{*}$ & degree & & & $M_{\odot} \mathrm{yr}^{-1}$ & & K \\
\hline MWC 480 & $2-20$ & $20^{\circ}$ & 5 & 3 & $3 \times 10^{-8}$ & 4 & 10000 \\
\hline IL Cep & $10-30$ & $30^{\circ}$ & 7 & 3 & $3 \times 10^{-8}$ & 3 & 10000 \\
\hline VV Ser & $5-15$ & $30^{\circ}$ & $3 / 2 / 1 / 1$ & 3 & $3.5 \times 10^{-8}$ & 2 & 10000 \\
\hline
\end{tabular}

Table 1: Disk wind model parameters

Theoretical study of the radiation of the different hydrogen lines from the accretion disk itself revealed its very little contribution to the total radiation of the $\operatorname{Br} \gamma$ line and more noticeable contribution to the radiation of the $\mathrm{H} \alpha$ line. Figure 4 demonstrates the $\mathrm{H} \alpha$ line profiles for the typical Herbig Ae star forming in the hot surface layers of the disk heated up to the temperatures of 10000 $\mathrm{K}$ by the hard radiation of the star. Details of modeling are present in the paper by Tambovtseva et al. (2016a). The line profiles are shown for inclinations $i$ from $0^{\circ}$ (the narrowest profile in Fig. 4) to $80^{\circ}$ (the widest profile). The line intensity is small compared to the observed lines by $\sim 10$ times 
and more but one cannot neglect it. It is necessary further investigations in this field, in particular, calculations of the thermal balance with a possible heating due to internal sources.

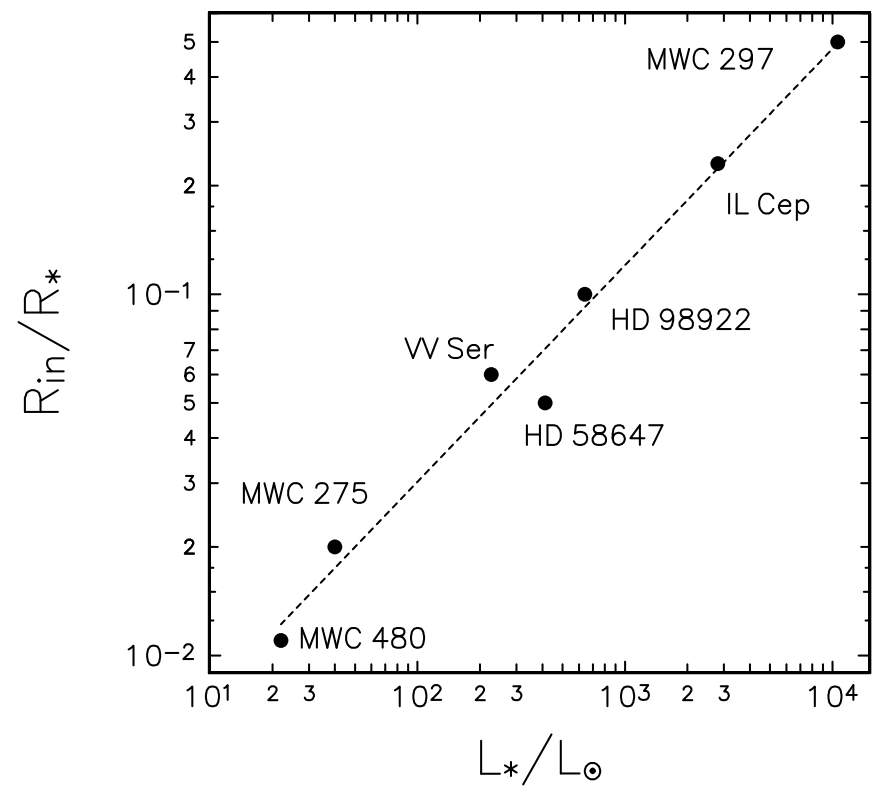

Figure 5: Diagram "Inner radius of the disk wind launching region vs stellar luminosity" for HAEBEs considered. Data for the star HD 58647 are taken from Kurosawa et al. 2016.

During our study of the Herbig AeBe stars we accumulated statistic data that permitted us to find some interesting consistency between parameters of the regions emitting in the hydrogen lines and parameters of the stars. One of these results is present in Fig. 5: one can see that the radius of the inner boundary of the disk wind launching region increases with the star luminosity. In other words, the launching region of the magneto-centrifugal disk wind is moved away with the increase in the luminosity. It can be probably explained with increasing effect of the radiation pressure onto the disk wind.

\section{Summary}

A magneto-centrifugal disk wind of Herbig AeBe stars is a dominant contributor to the hydrogen line radiation compared to the magnetosphere and inner gaseous accretion disk. A contribution of the accreting region into the hydrogen line emission is different for different stars. Magnetospheric accreting regions helps to explain triangle-like $\mathrm{Br} \gamma$ line profiles.

Interferometry together with spectroscopy provide constraints to the model parameters and give us an additional information about the star-plus-disk system.

\section{References}

[1] E. Alecian, G. A. Wade, C. Catala et al., A high-resolution spectropolarimetric survey of Herbig Ae/Be stars - I. Observations and measurements, MNRAS 429 (2013) 1001

[2] N. G. Beskrovnaya and M. A. Pogodin, Active phenomena in the circumstellar environment of the Herbig Ae star HD31648, A\&A 414 (2004) 955 
[3] R.D. Blandford and D.G. Payne, Hydromagnetic flows from accretion discs and the production of radio jets, MNRAS 199, (1982) 883

[4] A. Caratti o Garatti, L. V. Tambovtseva, R. Garcia Lopez et al., AMBER/VLTI high spectral resolution observations of the Bry emitting region in HD98922. A compact disc-wind launched from the inner disc region, $A \& A \mathbf{5 8 2}$ (2015)

[5] S.R. Cranmer, Turbulent-driven polar winds from T Tauri stars energized by magnetospheric accreton, ApJ 689 (2008) 316

[6] J. A. Eisner, J. D. Monnier, J. Woillez et al., Spatially and spectrally resolved hydrogen gas within 0.1 AU of T Tauri and Herbig Ae/Be stars, ApJ 718 (2010) 774

[7] J. A. Eisner, G. H. Rieke, M. J. Rieke et al., Time-monitoring observations of Bry emission from young stars, MNRAS 447 (2015) 202

[8] R. Garcia Lopez, L. V. Tambovtseva, D. Schertl et al. Probing the accretion-ejection connection with VLTI/AMBER High spectral resolution observations of the Herbig Ae star HD163296, A\&A 576 (2015) 84

[9] C. A. Grady, K. Hamaguchi, G. Schneider et al., Location the accretion footprint on a Herbig Ae star: MWC 480, ApJ 719 (2010) 1565

[10] V.P. Grinin, N.N. Kiselev, N. Kh. Minikulov et al., The investigations of zodiacal light of isolated AE Herbig stars with nonperiodic algol-type minima, Astrophys. and Space Sci. 186 (1991) 283

[11] V.P. Grinin, O.V. Kozlova, A. Natta et al. Optical spectra of five UX Orionistype stars, A\&A 379, (2001) 482

[12] V. P. Grinin and L. V. Tambovtseva, Disk Wind in the Radiation of Young Intermediate-Mass Stars. Astron. Rep. 55 (2011) 704

[13] V. P. Grinin, L. V. Tambovtseva, Potravnov I.P. and Mkrtichian D.E. Disk wind in the radiation of Herbig AeBe stars, in Proceedings of ASP Conf. Ser. The B[e] Phenomenon: Forty Years of Studies, (2016) in press

[14] L. Hartmann, R. Hewett and N. Calvet, Magnetospheric accretion models for T Tauri stars. 1: Balmer line profiles without rotation, ApJ 426 (1994) 669

[15] S. Hubrig, M.A. Pogodin R.V. Yudin et al. The magnetic field in the photospheric and circumstellar components of Herbig Ae stars, A\&A 463 (2007) 1039

[16] S. Hubrig, M. Schöller, I. Ilyin et al, Characterising the magnetic fields of the Herbig Ae/Be stars HD97048, HD150193, HD176386, and MWC480, A\&A 536 (2011) A45

[17] S. Hubrig, I. Ilyin, M. Schöller, and G. Lo Curto, HARPS spectropolarimetry of Herbig Ae/Be stars, Astron. Nachr. 334, (2013) 1093

[18] N.Z. Ismailov and O.V. Khalilov, Spectral Observations of AB Aur, Odessa Astronomical Publication 23 (2010) 49

[19] S. Kraus, K.-H. Hofmann, M. Benisty et al., The origin of hydrogen line emission for five Herbig Ae/Be stars spatially resolved by VLTI/AMBER spectro-interferometry, A\&A 489 (2008) 1157

[20] R. Kurosawa, T.J. Harries and N.H. Symington, On the formation of H $\alpha$ line emission around classical T Tauri stars, MNRAS 370 (2006) 580

[21] R. Kurosawa, A. Kreplin, G. Weigelt et al., Probing the wind-launching regions of the Herbig Be star HD 58647 with high spectral resolution interferometry, MNRAS 457 (2016) 2236 
[22] I. Mendigutía, N. Calvet, B. Montesinos et al., Accretion rates and accretion tracers of Herbig Ae/Be stars, A\&A 535 (2011) A99

[23] I. Mendigutía, S. Brittain, C. Eiroa et al., Accretion variability of Herbig Ae/Be stars observed by X-Shootter HD 31648 AND HD 163296 , ApJ 776 (2013) 44

[24] J. Muzerolle, N. Calvet and L. Hartmann, Emission-Line Diagnostics of T Tauri Magnetospheric Accretion. II. Improved Model Tests and Insights into Accretion Physics, ApJ 550 (2001) 944

[25] J. Muzerolle, P. D’Alessio, N. Calvet and L. Hartmann, Magnetospheres and disk accretion in Herbig Ae/Be stars, ApJ 617 (2004) 406

[26] L. V. Tambovtseva, V. P. Grinin, and O. V. Kozlova, Non-LTE models of accretion disks of UX Ori type stars, Astrophysics 42 (1999) 54

[27] L. V. Tambovtseva, V. P. Grinin, B. Rodgers, and O. V. Kozlova, Diagnostics for Accretion Disks Around UX Ori Stars Based on Balmer, Paschen, and Brackett Lines, Astron. Rep. 45 (2001) 442

[28] L.V. Tambovtseva, V.P. Grinin, and G. Weigelt, Hydrogen lines as a diagnostic tool for studying multicomponent emitting regions in hot young stars: magnetosphere, $X$-wind, and disk wind, $A \& A$ 562 (2014) A104

[29] L.V. Tambovtseva, V.P. Grinin, and G. Weigelt, A\&A Brackett gamma radiation from the inner gaseous accretion disk, magnetosphere, and disk wind region of Herbig AeBe stars, 590 (2016a) A97

[30] L. V. Tambovtseva, V. P. Grinin, I. S. Potravnov, and D. E.Mkrtichian, Disk Wind and Magnetospheric Accretion in Emission from the Herbig Ae Star MWC 480, Astronomy Letters 42 (2016b) 583

[31] G.A. Wade, E. Alecian, J. Grunhut, et al., in Proceedings of the Conference on Astronomical Polarimetry 2008: Science from Small to Large Telescopes, Quebec, Canada, 611 Ed. by P. Bastien and N. Manset, ASP Conf. Ser. (2009)

[32] G. Weigelt, V.P. Grinin, J. Groh et al. VLTI/AMBER spectro-interferometry of the Herbig Be star MWC 297 with spectral resolution 12 000, A\&A 527 (2011) A103

[33] C. Zanni and J. Ferreira, MHD simulations of accretion onto a dipolar magnetosphere I. Accretion curtains and the disk-locking paradigm, A\&A $\mathbf{5 0 8}$ (2009) 1117

[34] C. Zanni and J. Ferreira, MHD simulations of accretion onto a dipolar magnetosphere. II. Magnetospheric ejections and stellar spin-down, A\&A 550 (2013) A99 